Supplement of Atmos. Chem. Phys., 21, 7983-8002, 2021

https://doi.org/10.5194/acp-21-7983-2021-supplement

(C) Author(s) 2021. CC BY 4.0 License.

(c) (1)
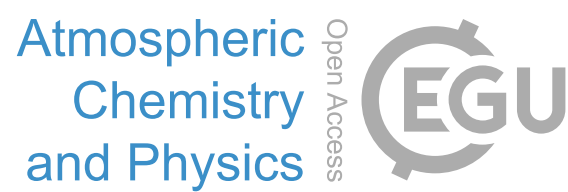

Supplement of

\title{
Aircraft measurements of aerosol and trace gas chemistry in the eastern North Atlantic
}

Maria A. Zawadowicz et al.

Correspondence to: John E. Shilling (john.shilling@pnnl.gov)

The copyright of individual parts of the supplement might differ from the article licence. 
There are several published methods of calibrating the AMS signal for the presence of MSA (Phinney et al., 2006, Huang et al., 2017, Ovadnevaite et al., 2011, Ge et al., 2012, Hodshire et al., 2019), but they all concentrate on the presence of a characteristic MSA marker, $\mathrm{CH}_{3} \mathrm{SO}_{2}{ }^{+}$. In addition, there are two additional minor characteristic markers, $\mathrm{CH}_{2} \mathrm{SO}_{2}{ }^{+}$and

$25 \mathrm{CH}_{4} \mathrm{SO}_{3}{ }^{+}$(Ge et al., 2012, Huang et al., 2017). A well-known difficulty in laboratory MSA calibrations is neutralization of the acidic aerosol by the presence of trace amount of ammonia in laboratory air and on surfaces (Hodshire et al., 2019). To overcome this difficulty, we adapt the approach detailed in Hodshire, et al. (2019) and first calibrate the AMS using the neutralized form of MSA, $\mathrm{NH}_{4} \mathrm{CH}_{3} \mathrm{SO}_{3}$. To prepare the calibration solution, MSA was neutralized with ammonium hydroxide. The final concentration of the calibration solution was $75 \mathrm{mM}$. The calibration solution was aerosolized with a

30 Collison-type atomizer (TSI 3076, Shoreview, MN) and dried using a Nafion dryer (5 slpm counter-flow). The resulting aerosol was size-selected with a Differential Mobility Analyzer (DMA) (TSI, Shoreview, MN) to 250, 300 and $350 \mathrm{~nm}$ (Supplementary Figure S2). The calibration was performed using simultaneous measurements of $\mathrm{CH}_{3} \mathrm{SO}_{3}{ }^{-}$ion concentration derived from Condensation Particle Counter (CPC) particle counts (TSI 3776, Shoreview, MN). In order to derive $\mathrm{CH}_{3} \mathrm{SO}_{3}^{-}$ ion concentration, density of $1.3 \mathrm{~g} / \mathrm{cm}^{3}$ and shape factor of 1 was assumed. AMS PTOF data were used to correct for 35 multiply charged particles. Resulting calibrations are shown in Supplementary Figure S2, along with a reference AMS mass spectrum of laboratory-generated neutralized MSA.

To quantify MSA, we use the combination of the three characteristic marker ions $\left(\mathrm{CH}_{2} \mathrm{SO}_{2}{ }^{+}+\mathrm{CH}_{3} \mathrm{SO}_{2}{ }^{+}+\mathrm{CH}_{4} \mathrm{SO}_{3}{ }^{+}\right)$. Using the standard AMS approach, (neutralized) MSA concentration is given by

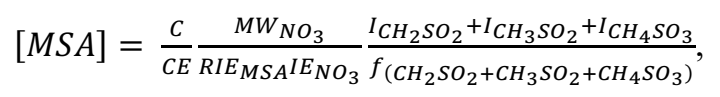

Where $\mathrm{C}$ is a proportionality constant accounting for instrument duty cycle, flow rate and unit conversions. CE is the AMS collection efficiency, RIEMSA is the ionization efficiency of (neutralized) MSA expressed proportionally to the ionization efficiency of nitrate $\left(\mathrm{IENO}_{3}\right)$, which is obtained by standard $\mathrm{AMS} \mathrm{NH} 4 \mathrm{NO}_{3}$ calibration. $\mathrm{I}_{\mathrm{x}}$ expresses the signal of marker ion $\mathrm{x}$

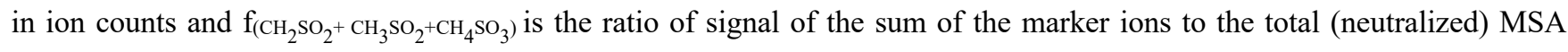
signal. If ion signal is expressed in nitrate-equivalent concentration units, the equation simplifies to

$$
[M S A]=\frac{1}{C E R I E_{M S A} f_{\left(\mathrm{CH}_{2} \mathrm{SO}_{2}+\mathrm{CH}_{3} \mathrm{SO}_{2}+\mathrm{CH}_{4} \mathrm{SO}_{3}\right)}}\left(I_{\mathrm{CH}_{2} \mathrm{SO}_{2}}+I_{\mathrm{CH}_{3} \mathrm{SO}_{2}}+I_{\mathrm{CH}_{4} \mathrm{SO}_{3}}\right)=\frac{C F}{C E}\left(I_{\mathrm{CH}_{2} \mathrm{SO}_{2}}+I_{\mathrm{CH}_{3} \mathrm{SO}_{2}}+\mathrm{I}_{\mathrm{CH}_{4} \mathrm{SO}_{3}}\right)
$$

50 Where $C F=\frac{1}{\mathrm{RIE}_{M S A} f_{\left(\mathrm{CH}_{2} \mathrm{SO}_{2}+\mathrm{CH}_{3} \mathrm{SO}_{2}+\mathrm{CH}_{4} \mathrm{SO}_{3}\right)}}$ is defined as the calibration factor and $\frac{C F}{C E}$ the slope in the laboratory calibration plot in Supplementary Figure S2, and has been determined to be $7.2 \pm 0.9$. 


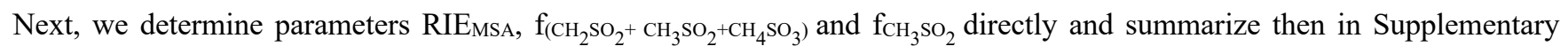
Table S2. The ratios of marker signals to the total (neutralized) MSA signal are derived from average neutralized MSA mass spectra (Supplementary Figure S2A). RIEMSA can be estimated with the ammonium balance method: using the previously 55 calibrated $\mathrm{RIENH}_{4}$ (4.1), RIEMSA is adjusted to balance the ammonium with its counter-ion, $\mathrm{CH}_{3} \mathrm{SO}_{3}$. This assumes complete neutralization of MSA in the AMS. Supplementary Table S2 compares these calibration parameters to previous literature estimates, showing a large spread in values, which underscores the importance of calibrating each AMS independently.

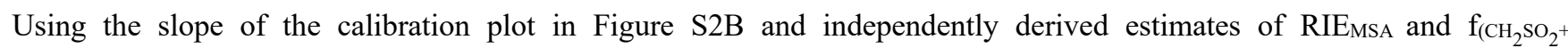
$\mathrm{CH}_{3} \mathrm{SO}_{2}+\mathrm{CH}_{4} \mathrm{SO}_{3}$, we can constrain the $\mathrm{CE}$ for the neutralized MSA used in laboratory calibrations as $0.61 \pm 0.07$. This is the

60 largest source of uncertainty in translating the calibrations to ambient measurements of MSA, as ambient MSA is more acidic than the neutralized laboratory MSA, which implies that the ambient particles less viscous, which is expected to affect $\mathrm{CE}$ (Middlebrook et al., 2012). If the CE of acidic form of MSA is assumed to be 1, the calibration factor CF is $4.4 \pm 0.5$, as shown in Figure S2C. This CE-corrected calibration factor is used to translate ACE-ENA AMS measurements to MSA concentrations.

65 Using the laboratory MSA calibrations, we also calculate the fraction of MSA signal assigned to organic and sulfate, $64 \%$ and 36\%, respectively (Supplementary Table S2). Some MSA ions, such as the three characteristic markers used in the calibration are organosulfates, which are classified as organic by the default AMS routine. 
70 Supplementary Table S1: Summary of ACE-ENA flights and vertical profiles.

\begin{tabular}{|c|c|c|c|c|c|c|}
\hline RF \# & Flight date & $\begin{array}{c}\text { Takeoff } \\
\text { (local time) }\end{array}$ & $\begin{array}{l}\text { Landing } \\
\text { (local time) }\end{array}$ & $\begin{array}{c}\text { Number of spiral } \\
\text { profiles }\end{array}$ & $\begin{array}{l}\text { Altitude range } \\
\text { (m) }\end{array}$ & $\begin{array}{c}\text { Location of } \\
\text { spiral profiles } \\
\text { (lat, lon) }\end{array}$ \\
\hline \multicolumn{7}{|c|}{ summer } \\
\hline \multirow{2}{*}{1} & \multirow{2}{*}{$6 / 21 / 17$} & \multirow{2}{*}{$11: 28$} & \multirow{2}{*}{$15: 10$} & \multirow{2}{*}{2} & \multirow{2}{*}{$100-3000$} & $39.23,-28.35$ \\
\hline & & & & & & $39.24,-28.37$ \\
\hline \multirow{4}{*}{2} & \multirow{4}{*}{$6 / 23 / 17$} & \multirow{4}{*}{$10: 46$} & \multirow{4}{*}{$14: 04$} & \multirow{4}{*}{4} & \multirow{4}{*}{$100-2500$} & $39.11,-28.03$ \\
\hline & & & & & & $39.79,-29.53$ \\
\hline & & & & & & $39.11,-28.01$ \\
\hline & & & & & & $39.18,-28.15$ \\
\hline \multirow{2}{*}{3} & \multirow{2}{*}{$6 / 25 / 17$} & \multirow{2}{*}{$10: 34$} & \multirow{2}{*}{$14: 25$} & \multirow{2}{*}{2} & \multirow{2}{*}{$100-1850$} & $39.11,-28.04$ \\
\hline & & & & & & $39.75,-29.27$ \\
\hline \multirow{2}{*}{4} & \multirow{2}{*}{$6 / 26 / 17$} & \multirow{2}{*}{$8: 30$} & \multirow{2}{*}{$12: 15$} & \multirow{2}{*}{2} & \multirow{2}{*}{$100-3300$} & $39.16,-28.19$ \\
\hline & & & & & & $39.26,-28.40$ \\
\hline \multirow{2}{*}{5} & \multirow{2}{*}{$6 / 28 / 17$} & \multirow{2}{*}{ 9:07 } & \multirow{2}{*}{$12: 29$} & \multirow{2}{*}{2} & $100 \quad 1000$ & $39.27,-28.31$ \\
\hline & & & & & $100-1000$ & $39.31,-27.80$ \\
\hline 6 & $6 / 29 / 17$ & $10 \cdot 30$ & $14 \cdot 34$ & 2 & $100-5000$ & $40.09,-28.06$ \\
\hline & דו & & & 2 & $100-3000$ & $40.11,-28.02$ \\
\hline 7 & $6 / 20 / 17$ & 0.27 & 12.16 & 0 & 1002000 & $39.30,-27.78$ \\
\hline 1 & $0 / 30 / 1 /$ & $9: 21$ & $15: 10$ & 2 & $100-2000$ & $39.30,-28.33$ \\
\hline 8 & $7 / 2 / 17$ & 10.27 & 14.48 & 2 & $100 \quad 2000$ & $39.11,-28.01$ \\
\hline 0 & $1 / 3 / 11 /$ & 10.51 & 14.40 & 2 & $100-2000$ & $39.10,-28.03$ \\
\hline & & & & & & $39.32,-28.30$ \\
\hline 0 & $7 / 1 / 17$ & 0.22 & 12.06 & 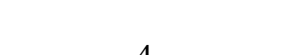 & 1002600 & $38.97-28.25$ \\
\hline (3) & 年 & 0.0 & 12.00 & $T$ & $100-2000$ & $39.31,-28.30$ \\
\hline & & & & & & $38.94,-28.24$ \\
\hline & & & & & & $39.35,-28.04$ \\
\hline 10 & $7 / 6 / 17$ & $8: 23$ & $11: 48$ & 3 & $100-1900$ & $39.37,-28.01$ \\
\hline & & & & & & $39.37,-28.02$ \\
\hline 11 & $7 / 7 / 17$ & 10.22 & 12.40 & 2 & $100 \quad 2200$ & $37.76,-27.14$ \\
\hline 11 & $1 / 1 / 17$ & 10.33 & 15.49 & 2 & $100-3500$ & $37.51,-26.85$ \\
\hline & & & & & & $40.02,-27.15$ \\
\hline 12 & $7 / 8 / 17$ & $8: 36$ & $12: 43$ & 4 & $100-3300$ & $39.60,-26.68$ \\
\hline & & & & & & $39.46,-26.51$ \\
\hline
\end{tabular}




\begin{tabular}{|c|c|c|c|c|c|c|}
\hline & & & & & & $39.82,-25.79$ \\
\hline \multirow{2}{*}{13} & \multirow{2}{*}{$7 / 11 / 17$} & \multirow{2}{*}{$10: 04$} & \multirow{2}{*}{$14: 04$} & \multirow{2}{*}{2} & \multirow{2}{*}{$100-2500$} & $39.32,-28.28$ \\
\hline & & & & & & $39.34,-28.26$ \\
\hline \multirow{2}{*}{14} & \multirow{2}{*}{$7 / 12 / 17$} & \multirow{2}{*}{$9: 00$} & \multirow{2}{*}{$13: 02$} & \multirow{2}{*}{2} & \multirow{2}{*}{$100-2100$} & $39.25,-28.06$ \\
\hline & & & & & & $39.27,-28.05$ \\
\hline \multirow{3}{*}{15} & \multirow{3}{*}{$7 / 13 / 17$} & \multirow{3}{*}{$8: 32$} & \multirow{3}{*}{$12: 55$} & \multirow{3}{*}{3} & \multirow{3}{*}{$100-2000$} & $39.33,-27.88$ \\
\hline & & & & & & $39.33,-27.85$ \\
\hline & & & & & & $39.02,-27.79$ \\
\hline \multirow{3}{*}{16} & \multirow{3}{*}{$7 / 15 / 17$} & \multirow{3}{*}{$10: 24$} & \multirow{3}{*}{$14: 24$} & \multirow{3}{*}{3} & \multirow{3}{*}{$100-2000$} & $39.21,-27.90$ \\
\hline & & & & & & $39.46,-27.97$ \\
\hline & & & & & & $39.41,-28.01$ \\
\hline \multirow{3}{*}{17} & \multirow{3}{*}{$7 / 17 / 17$} & \multirow{3}{*}{$9: 29$} & \multirow{3}{*}{$13: 28$} & & & $39.21,-28.37$ \\
\hline & & & & 3 & $100-2000$ & $39.39,-26.64$ \\
\hline & & & & & & $39.20,-25.87$ \\
\hline & & & & & & $39.34,-28.08$ \\
\hline 18 & $7 / 18 / 17$ & $8: 31$ & $12: 05$ & 3 & $100-2500$ & $39.30,-27.76$ \\
\hline & & & & & & $39.31,-28.28$ \\
\hline & & & & & & $39.17,-28.40$ \\
\hline & & & & & & $39.39,-27.90$ \\
\hline 19 & 7/19/17 & $8: 54$ & $12: 56$ & 5 & $100-3000$ & $39.38,-27.92$ \\
\hline & & & & & & $39.45,-27.59$ \\
\hline & & & & & & $39.79,-26.61$ \\
\hline & & & & & & $39.25,-27.72$ \\
\hline 20 & $7 / 20 / 17$ & $8: 31$ & $12: 11$ & 3 & $100-2500$ & $39.26,-27.70$ \\
\hline & & & & & & $39.35,-28.25$ \\
\hline & & & & nter & & \\
\hline & & & & & & $39.07,-28.37$ \\
\hline 21 & 1/19/18 & $11: 11$ & $15: 04$ & 3 & $100-2000$ & $39.07,-28.40$ \\
\hline & & & & & & $39.07,-28.42$ \\
\hline & & & & & & $38.81,-25.74$ \\
\hline 22 & $1 / 21 / 18$ & $8: 45$ & $12: 29$ & 3 & $100-2600$ & $38.82,-25.74$ \\
\hline & & & & & & $38.82,-25.70$ \\
\hline & & & & & & $39.31,-28.23$ \\
\hline 23 & $1 / 24 / 18$ & $11: 53$ & $15: 54$ & 3 & $100-2600$ & $39.30,-28.22$ \\
\hline & & & & & & $39.34,-28.18$ \\
\hline
\end{tabular}




\begin{tabular}{|c|c|c|c|c|c|c|}
\hline \multirow{4}{*}{24} & \multirow{4}{*}{$1 / 25 / 17$} & \multirow{4}{*}{ 10:01 } & \multirow{4}{*}{$13: 49$} & \multirow{4}{*}{4} & \multirow{4}{*}{$100-2600$} & $39.31,-27.86$ \\
\hline & & & & & & $39.23,-28.35$ \\
\hline & & & & & & $39.35,-27.88$ \\
\hline & & & & & & $39.35,-27.88$ \\
\hline \multirow{2}{*}{25} & \multirow{2}{*}{$1 / 26 / 18$} & \multirow{2}{*}{ 10:05 } & \multirow{2}{*}{$14: 03$} & \multirow{2}{*}{2} & \multirow{2}{*}{$100-2000$} & $38.01,-27.13$ \\
\hline & & & & & & $37.73,-27.90$ \\
\hline \multirow{2}{*}{26} & \multirow{2}{*}{$1 / 28 / 18$} & \multirow{2}{*}{$8: 38$} & \multirow{2}{*}{$12: 33$} & \multirow{2}{*}{2} & \multirow{2}{*}{$100-2700$} & $38.05,-27.17$ \\
\hline & & & & & & $38.32,-27.47$ \\
\hline \multirow{3}{*}{27} & \multirow{3}{*}{$1 / 29 / 18$} & \multirow{3}{*}{$8: 39$} & \multirow{3}{*}{$12: 32$} & \multirow{3}{*}{3} & \multirow{3}{*}{$100-2600$} & $38.97,-27.66$ \\
\hline & & & & & & $38.98,-27.67$ \\
\hline & & & & & & $39.35,-27.91$ \\
\hline \multirow{3}{*}{28} & \multirow{3}{*}{$1 / 30 / 18$} & \multirow{3}{*}{$8: 34$} & \multirow{3}{*}{$12: 50$} & & & $39.28,-27.65$ \\
\hline & & & & 3 & $100-2600$ & $39.44,-28.26$ \\
\hline & & & & & & $39.28,-27.64$ \\
\hline & & & & & & $39.28,-27.73$ \\
\hline 0 & 0 & $0-0$ & 18 & 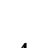 & مอ & $39.31,-28.26$ \\
\hline 29 & $2 / 1 / 10$ & 9.59 & 14.10 & 4 & $100-2100$ & $39.34,-28.23$ \\
\hline & & & & & & $39.37,-27.74$ \\
\hline 30 & $2 / 7 / 18$ & $16: 28$ & $18: 22$ & 0 & N/A & N/A \\
\hline & & & & & & $39.39,-27.96$ \\
\hline 31 & $2 / 8 / 18$ & $11: 54$ & $16: 04$ & 3 & $100-2300$ & $39.38,-27.91$ \\
\hline & & & & & & $39.36,-27.96$ \\
\hline & & & & & & $39.36,-27.82$ \\
\hline 22 & $2 / 0 / 10$ & 10.04 & 14.16 & 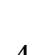 & $100 \quad 2500$ & $39.25,-28.34$ \\
\hline 32 & $2 / 9 / 18$ & $10: 04$ & $14: 16$ & 4 & $100-2500$ & $39.35,-27.79$ \\
\hline & & & & & & $39.32,-27.87$ \\
\hline & & & & & & $39.34,-27.79$ \\
\hline & & & & & & $39.31,-27.73$ \\
\hline 33 & $2 / 10 / 18$ & $11: 55$ & $15: 55$ & 5 & $100-3800$ & $39.31,-27.71$ \\
\hline & & & & & & $39.24,-28.35$ \\
\hline & & & & & & $39.22,-27.66$ \\
\hline & & & & & & $39.13,-28.05$ \\
\hline 24 & $2 / 11 / 10$ & 10.20 & 14.20 & 5 & $100 \quad 2000$ & $39.10,-28.02$ \\
\hline 34 & $2 / 11 / 18$ & $10: 20$ & $14: 20$ & 5 & $100-3900$ & $39.11,-28.03$ \\
\hline & & & & & & $39.44,-28.60$ \\
\hline
\end{tabular}




\begin{tabular}{|c|c|c|c|c|c|c|}
\hline & & & & & & $39.12,-28.04$ \\
\hline \multirow{3}{*}{35} & \multirow{3}{*}{$2 / 12 / 18$} & \multirow{3}{*}{$10: 05$} & \multirow{3}{*}{ 14:07 } & \multirow{3}{*}{3} & \multirow{3}{*}{$100-2500$} & $39.07,-28.42$ \\
\hline & & & & & & $39.06,-28.41$ \\
\hline & & & & & & $39.06,-28.38$ \\
\hline \multirow{3}{*}{36} & \multirow{3}{*}{$2 / 15 / 18$} & \multirow{3}{*}{$11: 59$} & \multirow{3}{*}{$16: 14$} & \multirow{3}{*}{3} & \multirow{3}{*}{$100-3700$} & $39.17,-27.98$ \\
\hline & & & & & & $39.64,-27.98$ \\
\hline & & & & & & $39.06,-28.04$ \\
\hline \multirow{4}{*}{37} & \multirow{4}{*}{$2 / 16 / 18$} & \multirow{4}{*}{$11: 54$} & \multirow{4}{*}{$16: 04$} & \multirow{4}{*}{4} & \multirow{4}{*}{$100-3700$} & $39.31,-27.81$ \\
\hline & & & & & & $39.28,-27.74$ \\
\hline & & & & & & $39.11,-28.10$ \\
\hline & & & & & & $39.30,-27.81$ \\
\hline \multirow{3}{*}{38} & \multirow{3}{*}{$2 / 18 / 18$} & \multirow{3}{*}{$11: 29$} & \multirow{3}{*}{$15: 41$} & \multirow{3}{*}{3} & \multirow{3}{*}{$100-3200$} & $39.48,-27.36$ \\
\hline & & & & & & $39.19,-28.02$ \\
\hline & & & & & & $39.47,-28.13$ \\
\hline \multirow{3}{*}{39} & \multirow{3}{*}{$2 / 19 / 18$} & \multirow{3}{*}{ 10:01 } & \multirow{3}{*}{$14: 09$} & \multirow{3}{*}{3} & \multirow{3}{*}{$100-4000$} & $39.32,-27.77$ \\
\hline & & & & & & $39.31,-27.72$ \\
\hline & & & & & & $39.35,-27.75$ \\
\hline
\end{tabular}


Supplementary Table S2: Calibration parameters for MSA in the PNNL AMS and comparisons to literature.

\begin{tabular}{|c|c|c|}
\hline Parameter & This work & Previous estimates if available \\
\hline MSAOrg $(\%)$ & $63.9 \pm 0.7$ & \\
\hline $\mathrm{MSASO}_{4}(\%)$ & $36.1 \pm 0.7$ & \\
\hline RIE $_{\text {MSA }}$ (UMR) & $1.09 \pm 0.02$ & \multirow{2}{*}{$\begin{array}{l}1.33 \text { (Willis et al., 2016), } \\
1.27 \text { (Huang et al., 2017), } \\
1.70 \pm 0.08 \text { (Hodshire et al., 2019) }\end{array}$} \\
\hline RIEMSA $_{\text {(HR) }}$ & $0.87 \pm 0.01$ & \\
\hline $\mathrm{f}_{\mathrm{CH}_{3} \mathrm{SO}_{2}(\%)}$ & $15.9 \pm 0.3$ & $\begin{array}{l}6.9 \text { (Phinney et al., 2006), } \\
9 \text { (Zorn et al., 2008), } \\
4 \text { (Schmale et al., 2013), } \\
9.7 \text { (Huang et al., 2015), } \\
12.4 \text { (Willis et al., 2016), } \\
4 \text { (Huang et al., 2017), } \\
7.9 \text { (Hodshire et al., 2019) }\end{array}$ \\
\hline 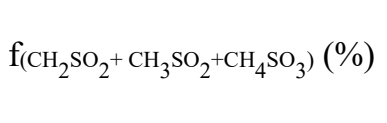 & $26.1 \pm 0.5$ & $\begin{array}{l}14.7 \text { (Ge et al., 2012), } \\
6.8 \pm 0.6 \text { (Huang et al., 2017) }\end{array}$ \\
\hline Calibration factor $\left(\mathrm{C}_{\mathrm{F}}\right)$ & $4.4 \pm 0.5$ & \\
\hline
\end{tabular}



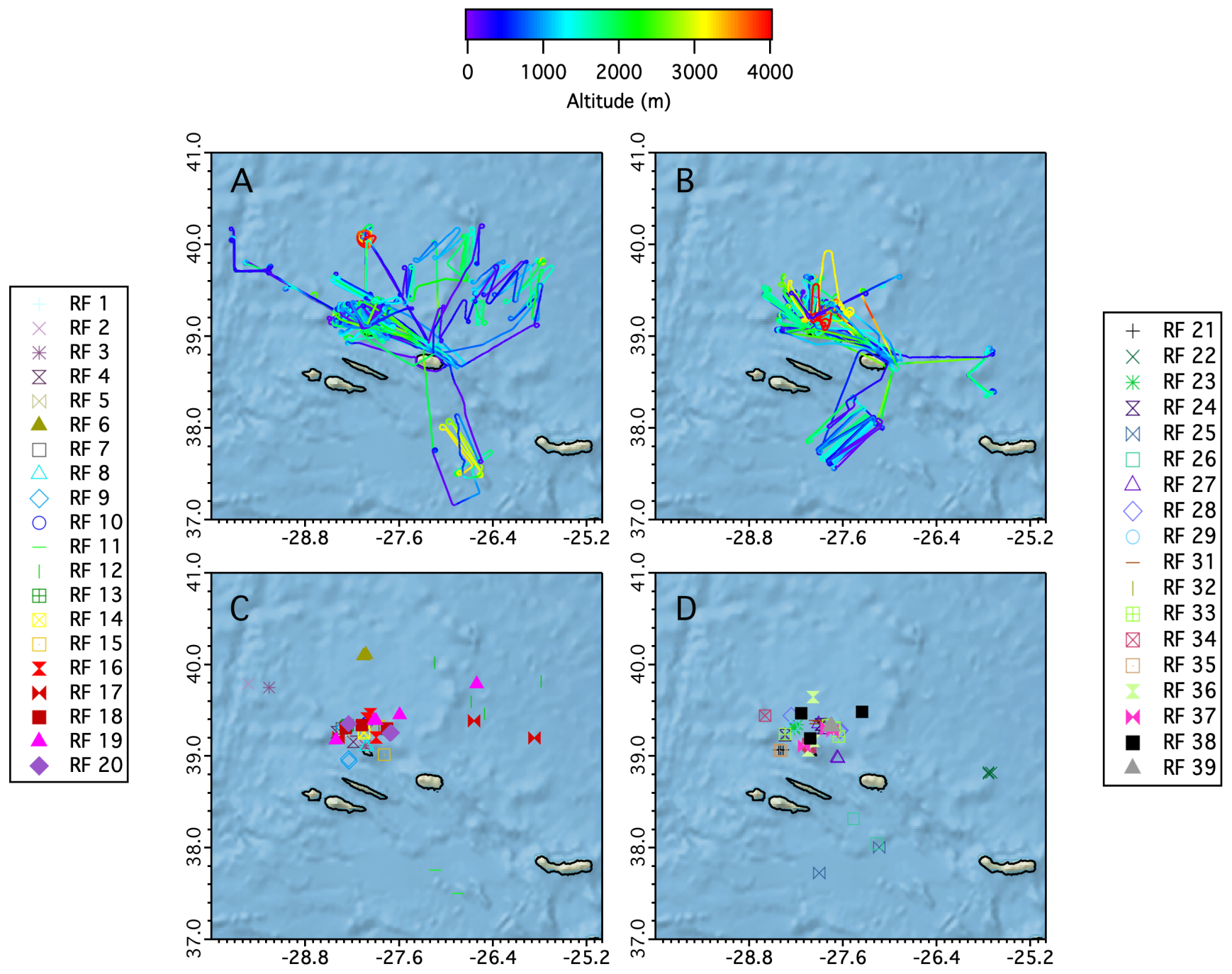

Supplementary Figure S1: Flight tracks for all G-1 flights carried out as a part of the ACE-ENA campaign. (A) All IOP 1 flight tracks. (B) All IOP 2 flight tracks. (C) Locations of spiral profiles for IOP 1. (D) Locations of spiral profiles for IOP 2.

80 The map was created using public domain map data on Natural Earth (naturalearthdata.com) and the GSHHG Database (ngdc.noaa.gov/mgg/shorelines/). 

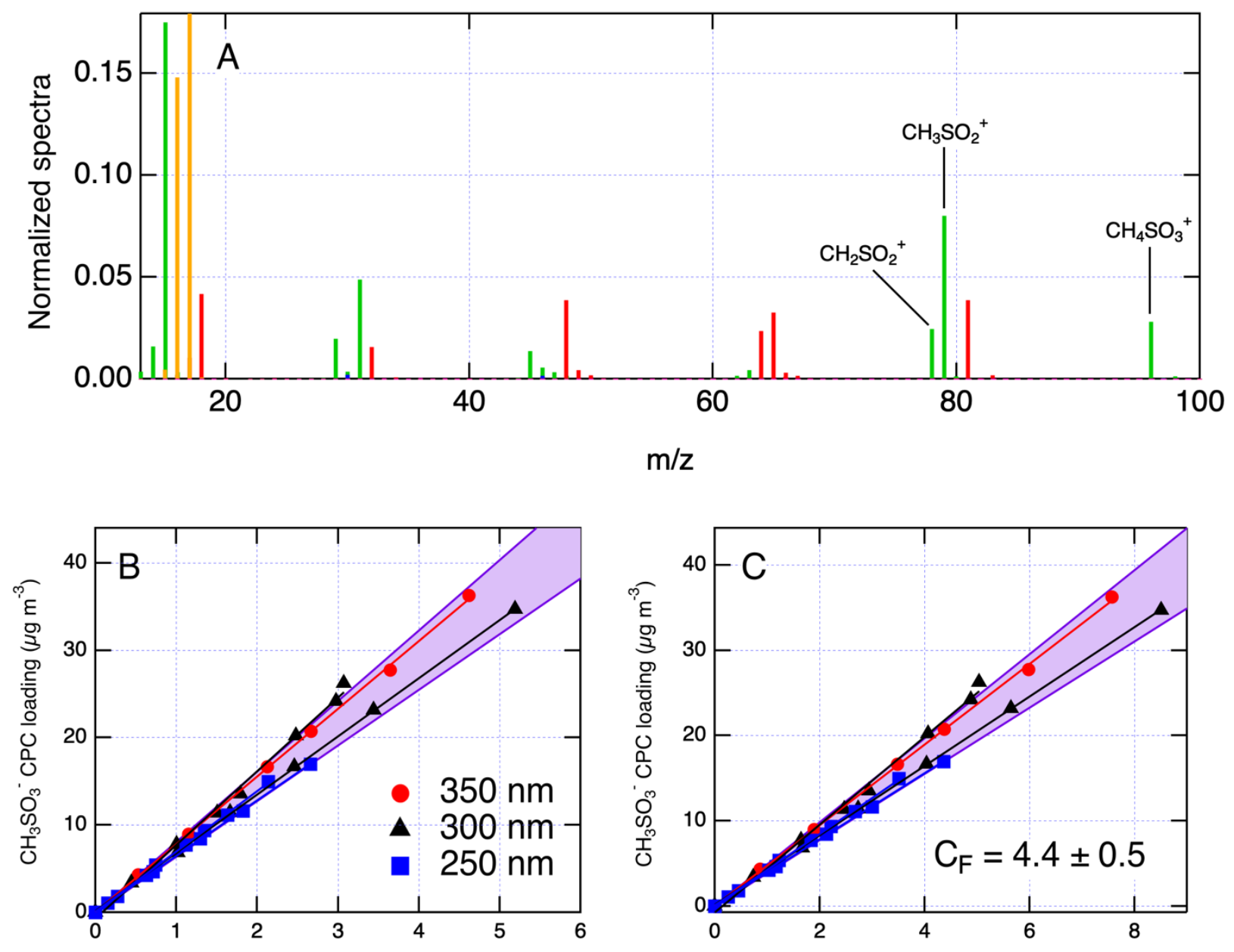

$$
\text { AMS } \mathrm{CH}_{2} \mathrm{SO}_{2}^{+}+\mathrm{CH}_{3} \mathrm{SO}_{2}^{+}+\mathrm{CH}_{4} \mathrm{SO}_{3}{ }^{+}\left(\mu \mathrm{g} \mathrm{m}^{-3}\right)
$$$$
\operatorname{AMS}\left(\mathrm{CH}_{2} \mathrm{SO}_{2}^{+}+\mathrm{CH}_{3} \mathrm{SO}_{2}^{+}+\mathrm{CH}_{4} \mathrm{SO}_{3}^{+}\right) / 0.61\left(\mu \mathrm{g} \mathrm{m}^{-3}\right)
$$

85 Supplementary Figure S2: Laboratory AMS calibrations for MSA. (A) Example spectrum of neutralized MSA used for the calibrations with key peaks labeled. (B) Calibrations using three different neutralized MSA sizes. Shaded region indicates uncertainty. 

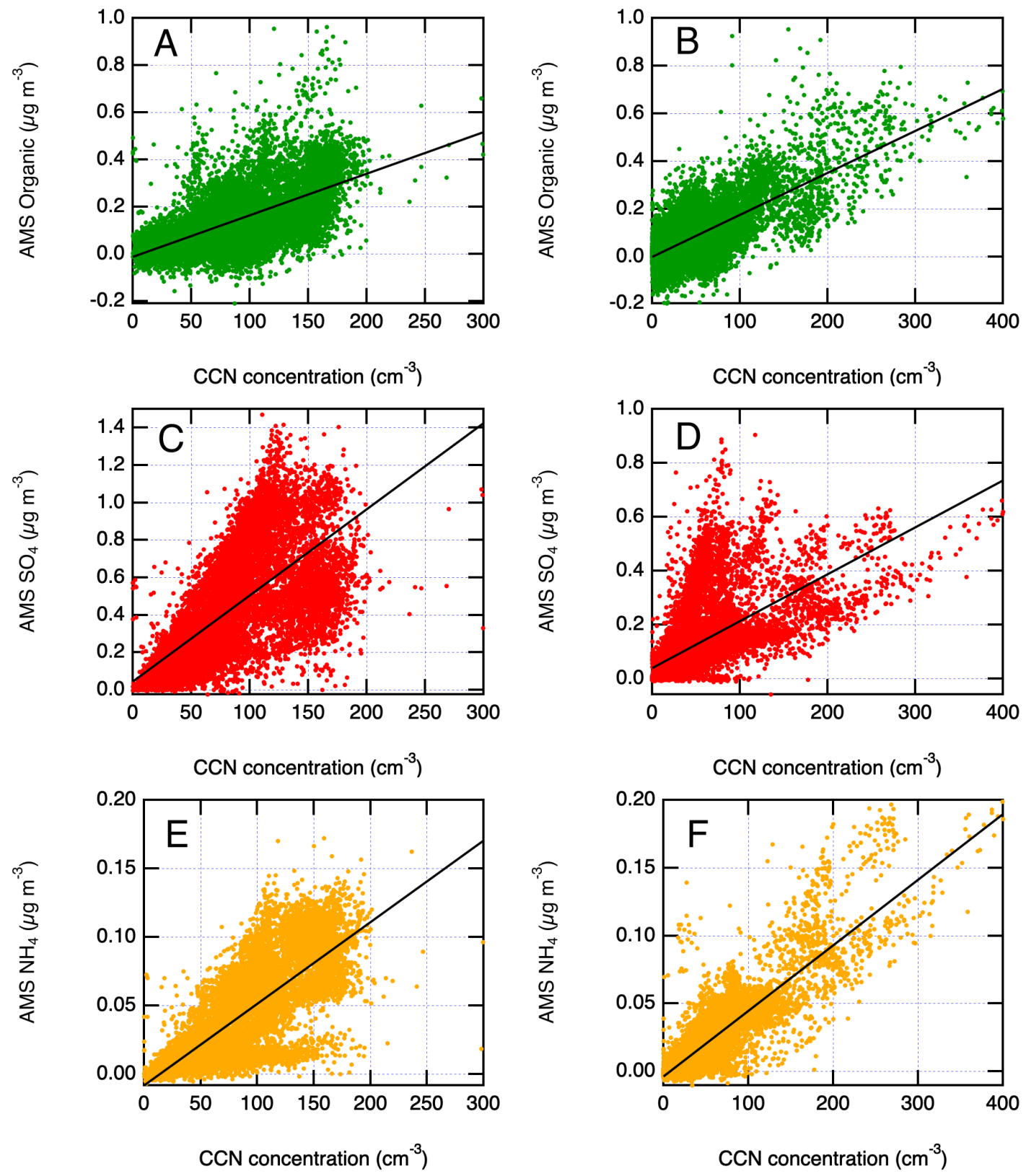

90 Supplementary Figure S3: Campaign-wide correlations between aerosol chemistry and CCN concentrations at $0.13 \%$ supersaturation. (A) AMS organic vs. $\mathrm{CCN}$ count, $<1000 \mathrm{~m}\left(\mathrm{R}^{2}=0.4\right)$. (B) AMS organic vs. CCN count, $>1000 \mathrm{~m}\left(\mathrm{R}^{2}=\right.$ 0.5). (C) AMS SO 4 vs. CCN count, $<1000 \mathrm{~m}\left(\mathrm{R}^{2}=0.5\right)$. (D) AMS SO 4 vs. $\mathrm{CCN}$ count, $>1000 \mathrm{~m}\left(\mathrm{R}^{2}=0.4\right)$. (E) $\mathrm{AMS} \mathrm{NH}_{4}$ vs. $\mathrm{CCN}$ count, $<1000 \mathrm{~m}\left(\mathrm{R}^{2}=0.6\right)$. (F) $\mathrm{AMS} \mathrm{NH}_{4}$ vs. $\mathrm{CCN}$ count, $>1000 \mathrm{~m}\left(\mathrm{R}^{2}=0.8\right)$. 

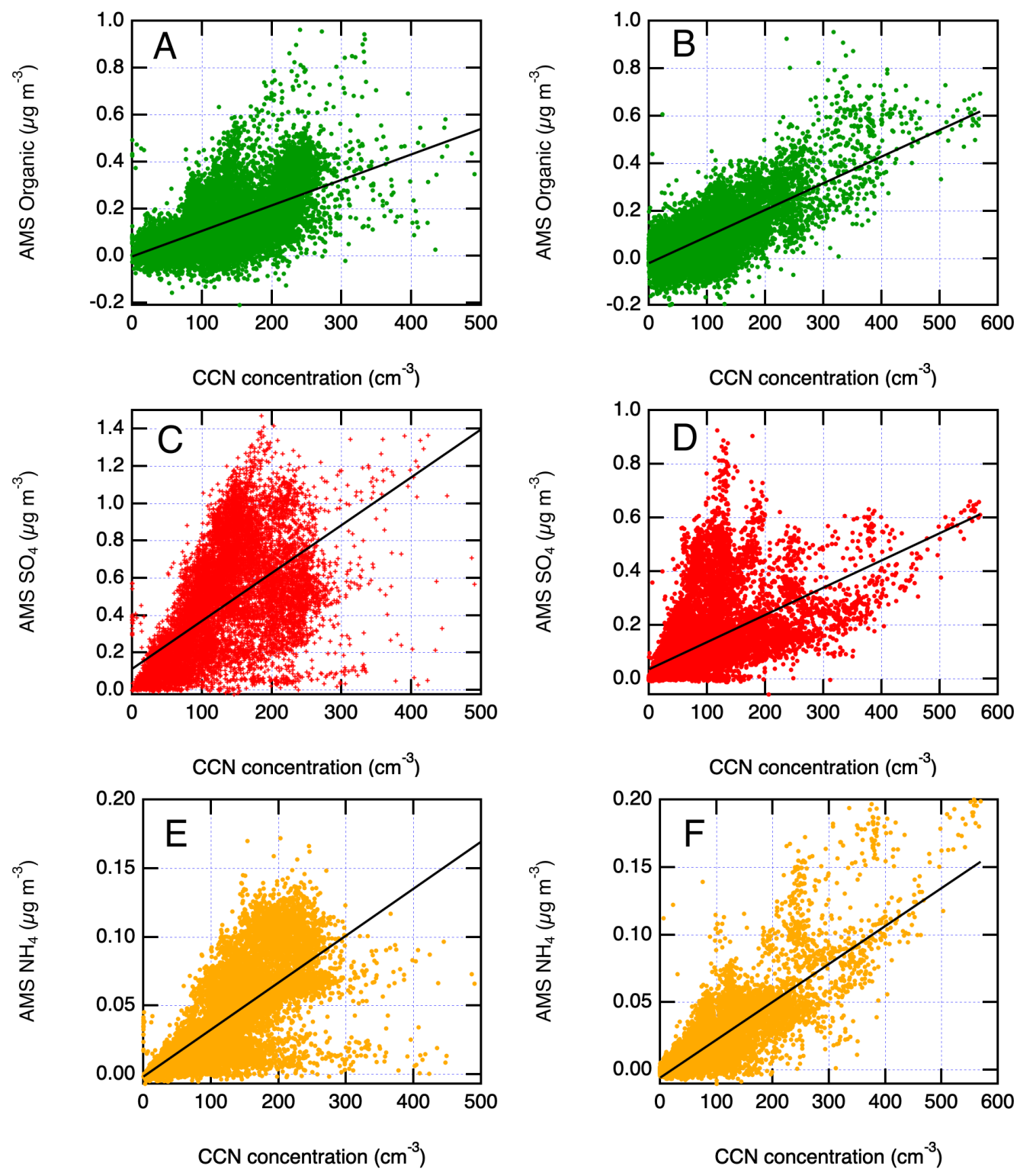

Supplementary Figure S4: Campaign-wide correlations between aerosol chemistry and CCN concentrations at $0.3 \%$ supersaturation. (A) AMS organic vs. $\mathrm{CCN}$ count, $<1000 \mathrm{~m}\left(\mathrm{R}^{2}=0.4\right)$. (B) AMS organic vs. CCN count, $>1000 \mathrm{~m}\left(\mathrm{R}^{2}=\right.$ 0.6). (C) $\mathrm{AMS} \mathrm{SO}_{4}$ vs. CCN count, $<1000 \mathrm{~m}\left(\mathrm{R}^{2}=0.4\right)$. (D) $\mathrm{AMS} \mathrm{SO}_{4}$ vs. $\mathrm{CCN}$ count, $>1000 \mathrm{~m}\left(\mathrm{R}^{2}=0.3\right)$. (E) $\mathrm{AMS} \mathrm{NH}_{4}$ vs. $\mathrm{CCN}$ count, $<1000 \mathrm{~m}\left(\mathrm{R}^{2}=0.5\right)$. (F) $\mathrm{AMS} \mathrm{NH}_{4}$ vs. $\mathrm{CCN}$ count, $>1000 \mathrm{~m}\left(\mathrm{R}^{2}=0.7\right)$. 

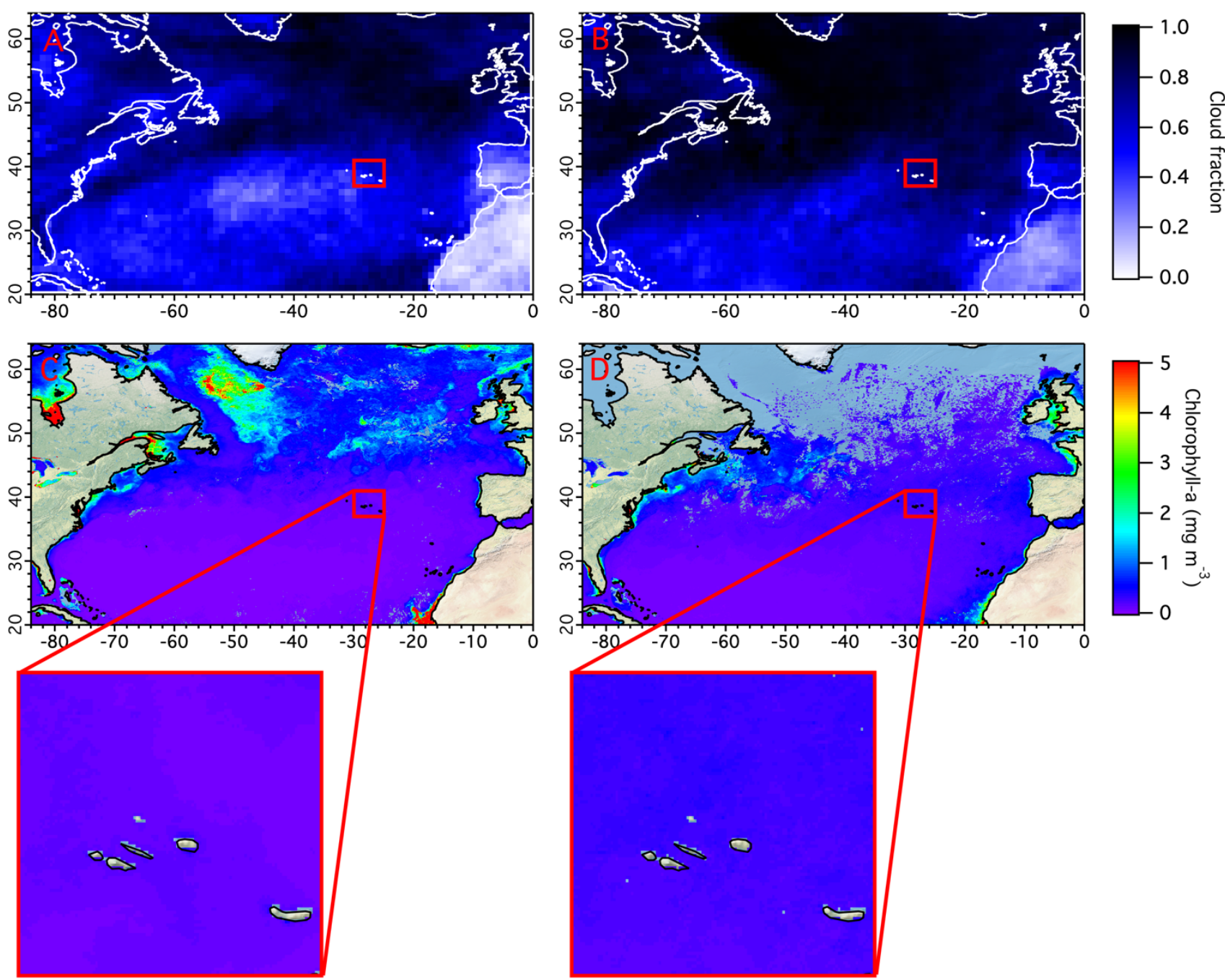

Supplementary Figure S5: Satellite measurements of relevant cloud and biogeochemical parameters during ACE-ENA. (A) MODIS Aqua time-averaged map of cloud fraction from cloud mask (count of lowest 2 clear sky confidence levels, cloudy

105 and probably cloudy divided by the total count). Mean of daily mean, $1^{\circ}$ resolution, from $06 / 21 / 2017$ to $07 / 20 / 2017$ (IOP 1 ) (Platnick, 2015). (B) Same as (A) but from 01/19/2018 to 02/19/2018 (IOP 2). (C) MODIS Aqua time-averaged map of chlorophyll-a concentration. Mean of 8-day means, 4 km resolution, 06/18/2017 to 07/28/2017 (IOP 1) (NASA Goddard Space Flight Center, 2018). (D) Same as (C) but from 01/17/2018 to 02/26/2018 (IOP 2). Red box indicates the Azores. The map was created using public domain map data on Natural Earth (naturalearthdata.com) and the GSHHG Database

110 (ngdc.noaa.gov/mgg/shorelines/). 


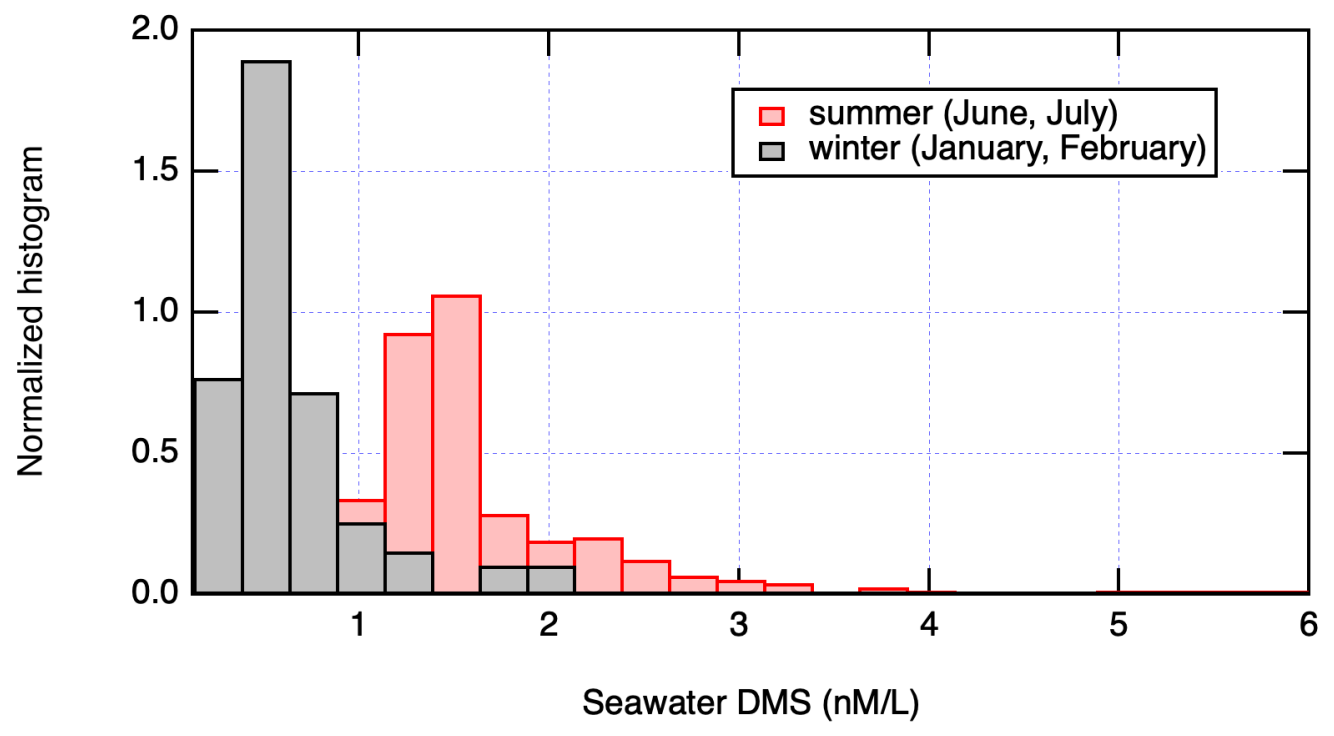

Supplementary Figure S6: The Global Surface Seawater DMS Database (saga.pmel.noaa.gov/dms/) (Kettle et al., 1999) was used to find surface seawater DMS measurements during January and February $(\mathrm{n}=78)$ and June and July $(\mathrm{n}=293)$ in 115 latitudes between $30.7^{\circ} \mathrm{N}$ and $48.4^{\circ} \mathrm{N}$ and longitudes between $12.6^{\circ} \mathrm{W}$ and $38.4^{\circ} \mathrm{W}$ (enclosing the Azores). 

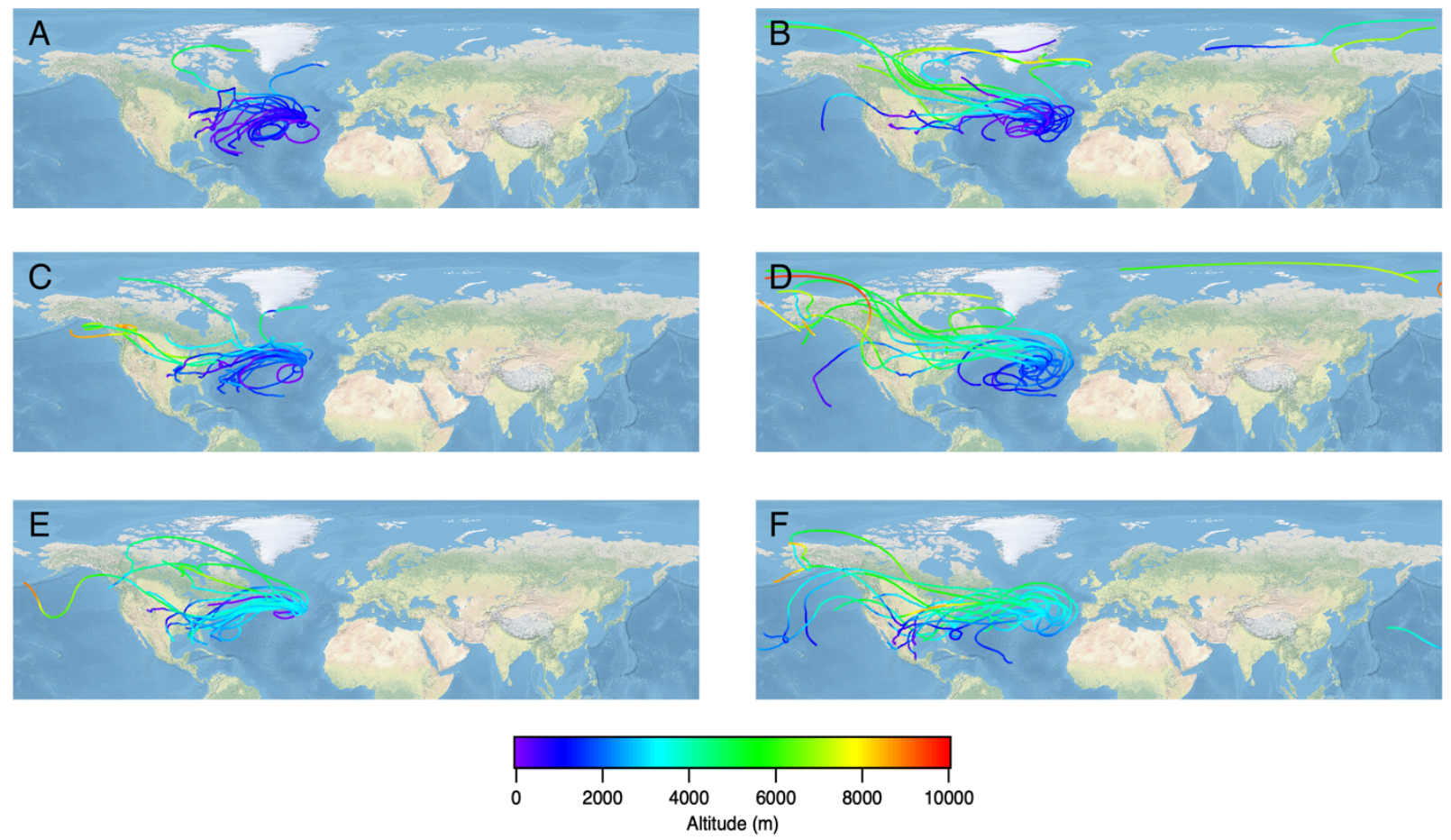

Supplementary Figure S7: HYSPLIT 14-day back-trajectories for each flight. The starting point is the ENA ARM site. (A)

$1201000 \mathrm{~m}$ starting altitude, IOP 1. (B) $1000 \mathrm{~m}$ starting altitude, IOP 2. (C) $2000 \mathrm{~m}$ starting altitude, IOP 1. (D) $2000 \mathrm{~m}$ starting altitude, IOP 2. (E) $3000 \mathrm{~m}$ starting altitude, IOP 1. (F) $3000 \mathrm{~m}$ starting altitude, IOP 2. The map was created using public domain map data on Natural Earth (naturalearthdata.com) and the GSHHG Database (ngdc.noaa.gov/mgg/shorelines/). 

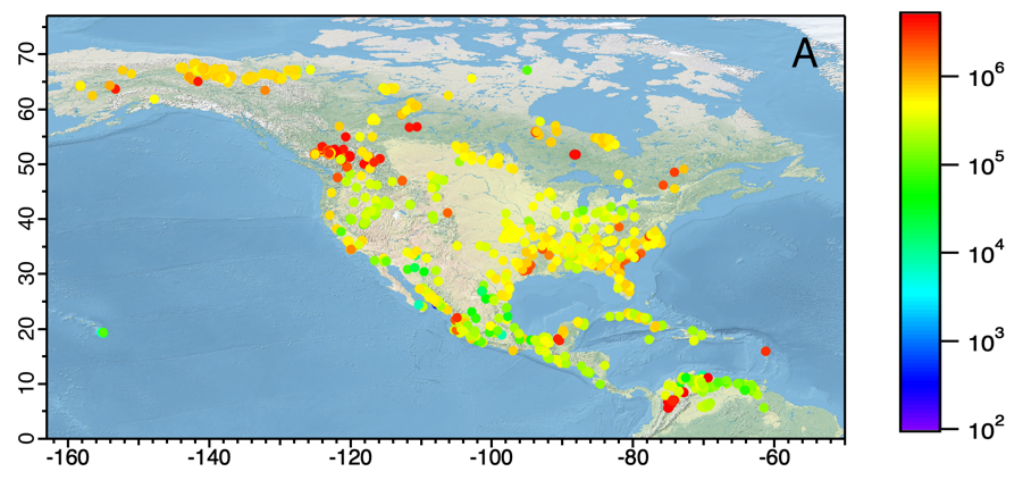

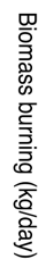
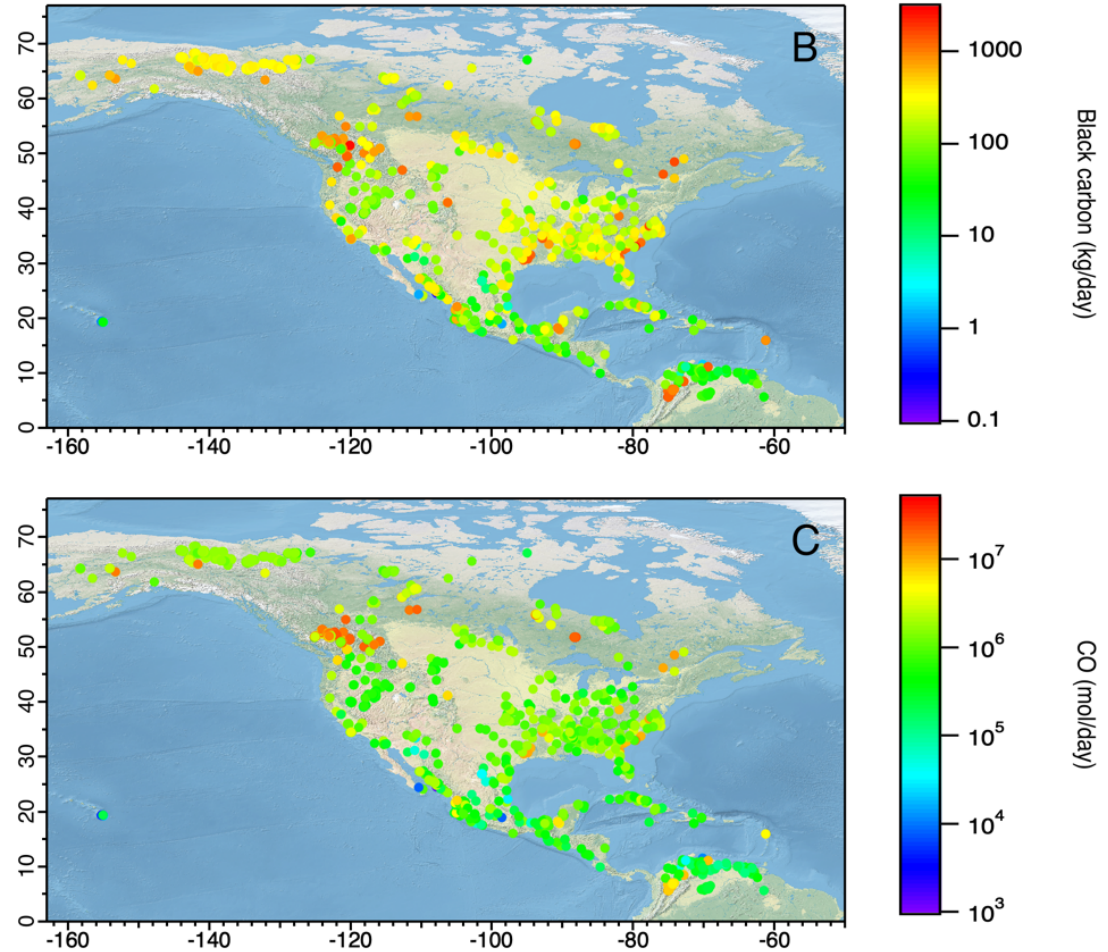

응

125 Supplementary Figure S8: Fire emissions during July 12 - 15, 2017 estimated using the Fire Inventory from NCAR (FINN) (Wiedinmyer et al., 2011). (A) Biomass burning emissions. (B) Black carbon emissions. (C) CO emissions. The map was created using public domain map data on Natural Earth (naturalearthdata.com) and the GSHHG Database (ngdc.noaa.gov/mgg/shorelines/). 

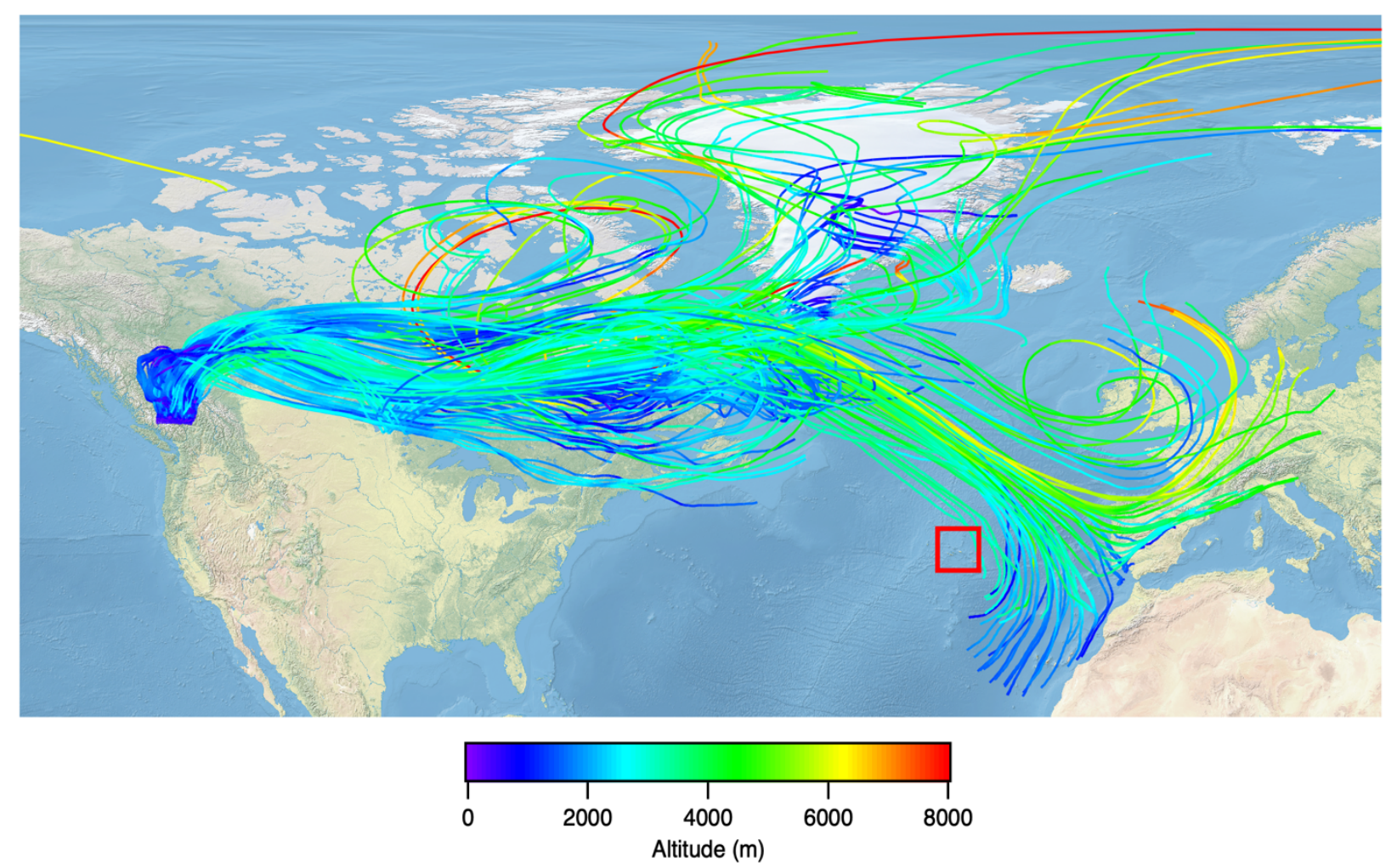

Supplementary Figure S9: HYSPLIT trajectory analysis for the case study of RF \#19 (July 19, 2017). A matrix of 121 10day forward-trajectories was started from an evenly spaced grid bounded by $(53.4 \mathrm{~N}, 125 \mathrm{~W}),(53.4 \mathrm{~N}, 121 \mathrm{~W}),(51 \mathrm{~N}, 125$ $\mathrm{W})$ and $(51 \mathrm{~N}, 121 \mathrm{~W})$ at $500 \mathrm{~m}$ altitude. GDAS 0.5 degree meteorology and isentropic vertical motion were used. The red box indicates the location of the Azores. The map was created using public domain map data on Natural Earth 135 (naturalearthdata.com) and the GSHHG Database (ngdc.noaa.gov/mgg/shorelines/). 
A

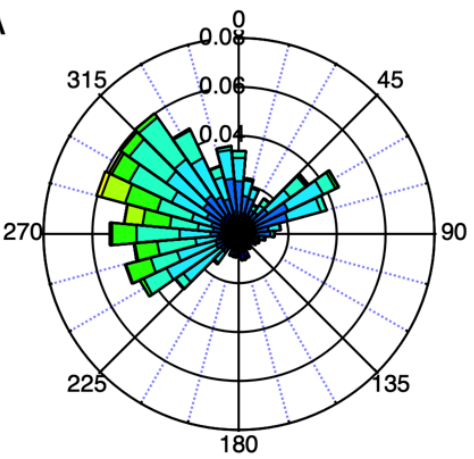

C

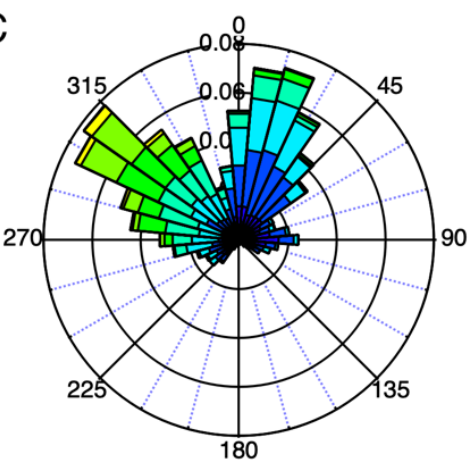

$E$

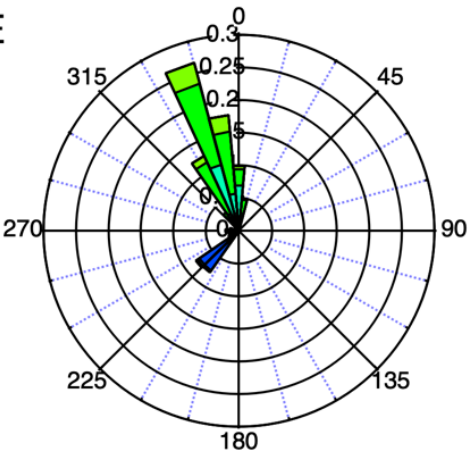

B

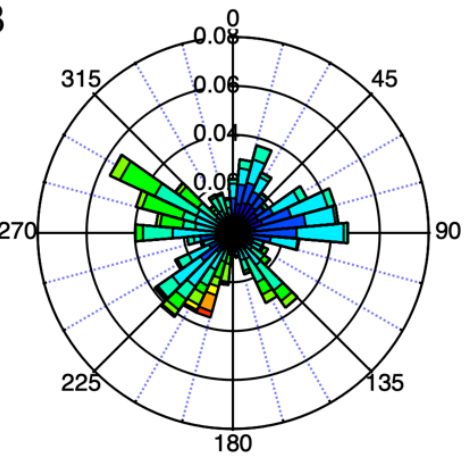

D

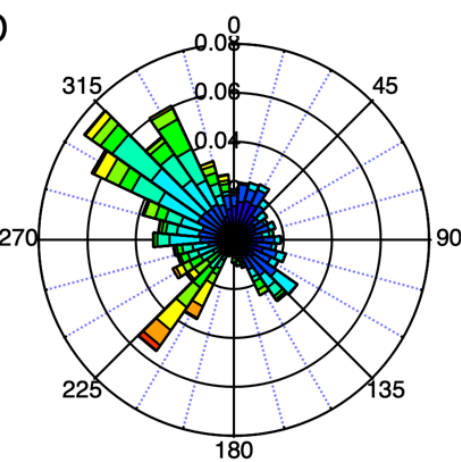

$\mathrm{F}$

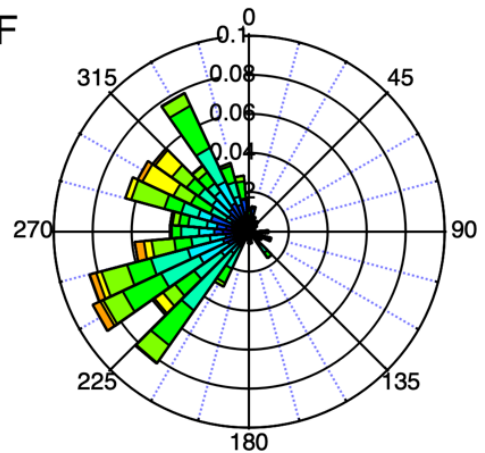

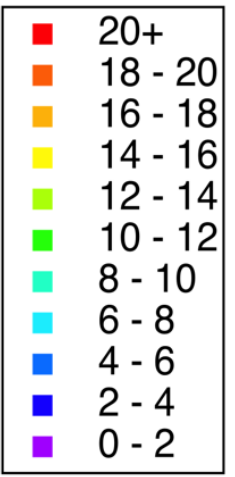

$0-2$

Supplementary Figure S10: Wind roses plotted using data from the G-1 AIMMS-20 probe during all ACE-ENA flights. (A) $<1000 \mathrm{~m}$ altitude, IOP 1. (B) < $1000 \mathrm{~m}$ altitude, IOP 2. (C) $1000 \mathrm{~m}-3000 \mathrm{~m}$ altitude, IOP 1. (D) $1000 \mathrm{~m}-3000 \mathrm{~m}$ 140 altitude, IOP 2. (E) > $3000 \mathrm{~m}$ altitude, IOP 1. (F) > $3000 \mathrm{~m}$ altitude, IOP 2. 

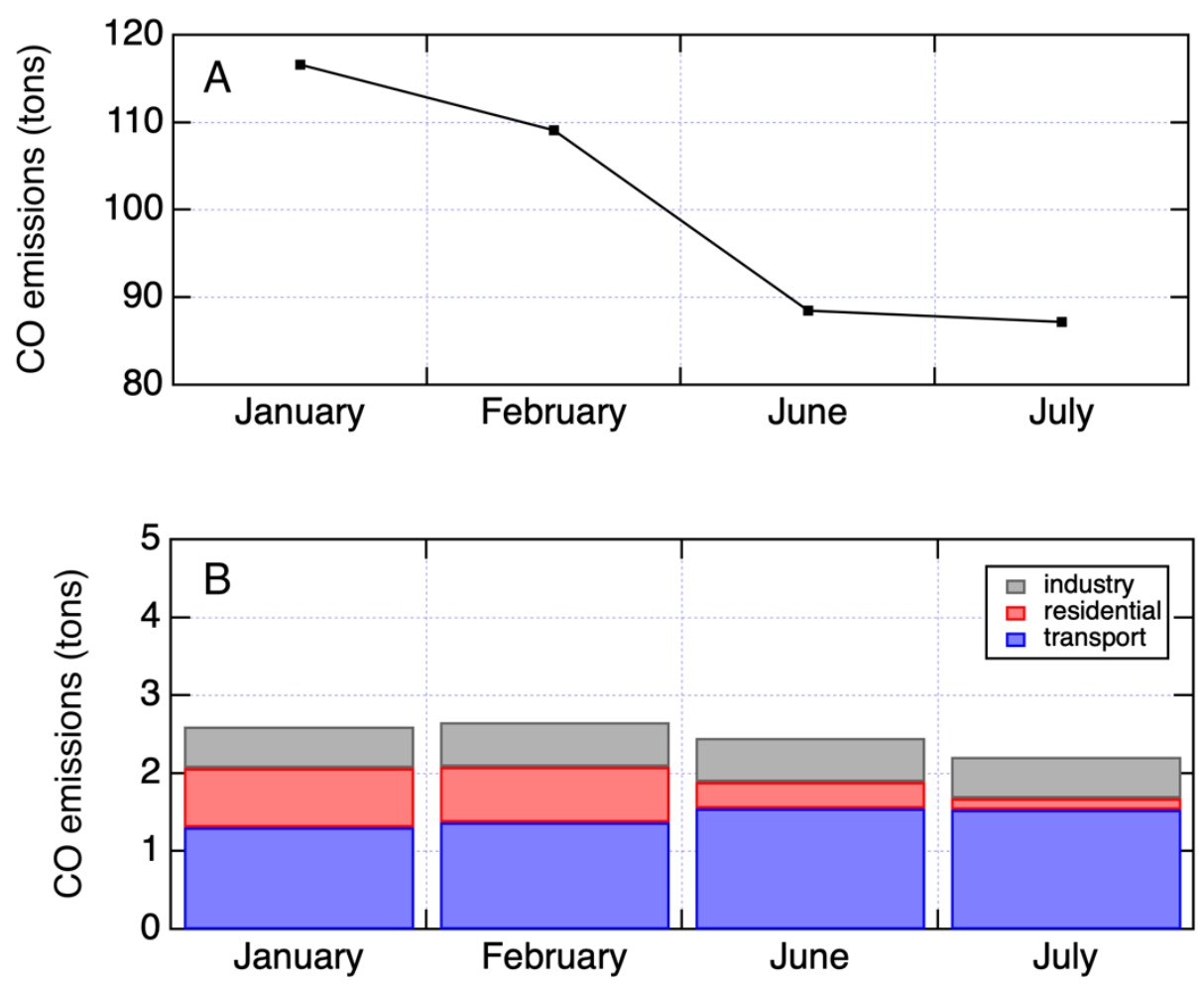

Supplementary Figure S11: EDGAR-HTAP V2 (https://edgar.jrc.ec.europa.eu/htap_v2/) gridded emissions inventory (Janssens-Maenhout et al., 2015) was used to investigate CO emissions from the Azores during the four months of ACE145 ENA study. Monthly gridmaps $0.1 \times 0.1$ for 2010 were used for this plot. (A) CO emissions from the energy sector. Data from one gridpoint at $38.7^{\circ} \mathrm{N}, 27.2^{\circ} \mathrm{W}$ is available for the Azores. (B) $\mathrm{CO}$ emissions from industry, residential and transport sectors. Data from 87 gridpoints are available for the Azores $\left(34^{\circ} \mathrm{N}-42^{\circ} \mathrm{N}, 20^{\circ} \mathrm{W}-35^{\circ} \mathrm{W}\right)$, averages by sector are reported in the plot. 
Ge, X., Zhang, Q., Sun, Y., Ruehl, C. R., and Setyan, A.: Effect of aqueous-phase processing on aerosol chemistry and size distributions in Fresno, California, during wintertime, Environmental Chemistry, 9, 10.1071/en11168, 2012.

Hodshire, A. L., Campuzano-Jost, P., Kodros, J. K., Croft, B., Nault, B. A., Schroder, J. C., Jimenez, J. L., and Pierce, J. R.: The potential role of methanesulfonic acid (MSA) in aerosol formation and growth and the associated radiative forcings,

155 Atmospheric Chemistry and Physics, 19, 3137-3160, 10.5194/acp-19-3137-2019, 2019.

Huang, D. D., Li, Y. J., Lee, B. P., and Chan, C. K.: Analysis of organic sulfur compounds in atmospheric aerosols at the HKUST supersite in Hong Kong using HR-ToF-AMS, Environ Sci Technol, 49, 3672-3679, 10.1021/es5056269, 2015.

Huang, S., Poulain, L., van Pinxteren, D., van Pinxteren, M., Wu, Z., Herrmann, H., and Wiedensohler, A.: Latitudinal and Seasonal Distribution of Particulate MSA over the Atlantic using a Validated Quantification Method with HR-ToF-AMS,

160 Environ Sci Technol, 51, 418-426, 10.1021/acs.est.6b03186, 2017.

Janssens-Maenhout, G., Crippa, M., Guizzardi, D., Dentener, F., Muntean, M., Pouliot, G., Keating, T., Zhang, Q., Kurokawa, J., Wankmüller, R., Denier van der Gon, H., Kuenen, J. J. P., Klimont, Z., Frost, G., Darras, S., Koffi, B., and Li, M.: HTAP_v2.2: a mosaic of regional and global emission grid maps for 2008 and 2010 to study hemispheric transport of air pollution, Átmospheric Chemistry and Physics, 15, 11411-11432, 10.5194/acp-15-11411-2015, 2015.

165 Kettle, A. J., Andreae, M. O., Amouroux, D., Andreae, T. W., Bates, T. S., Berresheim, H., Bingemer, H., Boniforti, R., Curran, M. A. J., DiTullio, G. R., Helas, G., Jones, G. B., Keller, M. D., Kiene, R. P., Leck, C., Levasseur, M., Malin, G., Maspero, M., Matrai, P., McTaggart, A. R., Mihalopoulos, N., Nguyen, B. C., Novo, A., Putaud, J. P., Rapsomanikis, S., Roberts, G., Schebeske, G., Sharma, S., Simó, R., Staubes, R., Turner, S., and Uher, G.: A global database of sea surface dimethylsulfide (DMS) measurements and a procedure to predict sea surface DMS as a function of latitude, longitude, and

170 month, Global Biogeochemical Cycles, 13, 399-444, 10.1029/1999gb900004, 1999.

Middlebrook, A. M., Bahreini, R., Jimenez, J. L., and Canagaratna, M. R.: Evaluation of Composition-Dependent Collection Efficiencies for the Aerodyne Aerosol Mass Spectrometer using Field Data, Aerosol Science and Technology, 46, 258-271, 10.1080/02786826.2011.620041, 2012.

NASA Goddard Space Flight Center, O. E. L., Ocean Biology Processing Group,: Moderate-resolution Imaging

175 Spectroradiometer (MODIS) Aqua Chlorophyll Data; 2018 Reprocessing, in, NASA OB.DAAC, Greenbelt, MD, USA, 2018.

Ovadnevaite, J., O'Dowd, C., Dall'Osto, M., Ceburnis, D., Worsnop, D. R., and Berresheim, H.: Detecting high contributions of primary organic matter to marine aerosol: A case study, Geophysical Research Letters, 38, n/a-n/a, 10.1029/2010gl046083, 2011.

180 Phinney, L., Richard Leaitch, W., Lohmann, U., Boudries, H., Worsnop, D. R., Jayne, J. T., Toom-Sauntry, D., Wadleigh, M., Sharma, S., and Shantz, N.: Characterization of the aerosol over the sub-arctic north east Pacific Ocean, Deep Sea Research Part II: Topical Studies in Oceanography, 53, 2410-2433, 10.1016/j.dsr2.2006.05.044, 2006.

Platnick, S.: MODIS Atmosphere L3 Daily Product, in, edited by: System, N. M. A. P., Goddard Space Flight Center, USA, 2015.

185 Schmale, J., Schneider, J., Nemitz, E., Tang, Y. S., Dragosits, U., Blackall, T. D., Trathan, P. N., Phillips, G. J., Sutton, M., and Braban, C. F.: Sub-Antarctic marine aerosol: dominant contributions from biogenic sources, Atmospheric Chemistry and Physics, 13, 8669-8694, 10.5194/acp-13-8669-2013, 2013.

Wiedinmyer, C., Akagi, S. K., Yokelson, R. J., Emmons, L. K., Al-Saadi, J. A., Orlando, J. J., and Soja, A. J.: The Fire INventory from NCAR (FINN): a high resolution global model to estimate the emissions from open burning, Geoscientific

190 Model Development, 4, 625-641, 10.5194/gmd-4-625-2011, 2011.

Willis, M. D., Burkart, J., Thomas, J. L., Köllner, F., Schneider, J., Bozem, H., Hoor, P. M., Aliabadi, A. A., Schulz, H., Herber, A. B., Leaitch, W. R., and Abbatt, J. P. D.: Growth of nucleation mode particles in the summertime Arctic: a case study, Atmospheric Chemistry and Physics, 16, 7663-7679, 10.5194/acp-16-7663-2016, 2016.

Zorn, S. R., Drewnick, F., Schott, M., Hoffmann, T., and Borrmann, S.: Characterization of the South Atlantic marine 195 boundary layer aerosol using an aerodyne aerosol mass spectrometer, Atmospheric Chemistry and Physics, 8, 4711-4728, 10.5194/acp-8-4711-2008, 2008. 